\title{
ALL MAXIMAL COMMUTATIVE SUBALGEBRAS OCCUR IN $L(X)$ UNCOUNTABLY MANY TIMES
}

\author{
WIESŁAW ŻELAZKO
}

\begin{abstract}
We show that for every Banach space $X, \operatorname{dim} X>1$, every maximal commutative subalgebra of $L(X)$ has uncountably many copies between maximal commutative subalgebras of $L(X)$. Answering to a question of Aleksander Pełczyński, we show also that for an arbitrary infinite dimensional Banach space $X$ there are at least countably many multiplications making of $X$ a commutative unital Banach algebra.
\end{abstract}

Keywords: algebra of Banach space operators, maximal commutative subalgebra, multiplications on Banach spaces.

Theorem. Let $X$ be a Banach space, $\operatorname{dim} X>1$. Let $\mathcal{A}$ be a maximal commutative subalgebra of $L(X)$. Then $L(X)$ contains uncountably many copies of $\mathcal{A}$ (i.e. maximal commutative subalgebras of $L(X)$ isomorphic to $\mathcal{A})$.

Proof. Denote by $\mathfrak{A}(X)$ the family of all maximal commutative subalgebras of $L(X)$ and let $\mathcal{A} \in \mathfrak{A}(X)$. It is easy to see that for any $Q \in L(X)$ the algebra

$$
e^{Q} \mathcal{A} e^{-Q}
$$

is in $\mathfrak{A}(X)$ and is (topologically) isomorphic to $\mathcal{A}$. For $Q$ as above consider the set

$$
G_{Q}(\mathcal{A})=\left\{t \in \mathbb{R}: e^{t Q} \mathcal{A} e^{-t Q}=\mathcal{A}\right\} .
$$

First we show that it is a closed subgroup of the (additive) group $\mathbb{R}$. In fact, the relation

$$
e^{t Q} \mathcal{A} e^{-t Q}=\mathcal{A}
$$

implies

$$
e^{-t Q} \mathcal{A} e^{t Q}=\mathcal{A}
$$

2010 Mathematics Subject Classification: primary: 47L10; secondary: 46H10, 46J05 
and so $t \in G_{Q}(\mathcal{A})$ implies $-t \in G_{Q}(\mathcal{A})$. If $t_{1}, t_{2} \in G_{Q}(\mathcal{A})$, then the formula (1) implies

$$
\mathcal{A}=e^{t_{1} Q} \mathcal{A} e^{-t_{1} Q}=e^{t_{2} Q} e^{t_{1} Q} \mathcal{A} e^{-t_{1} Q} e^{-t_{2} Q}=e^{\left(t_{1}+t_{2}\right) Q} \mathcal{A} e^{-\left(t_{1}+t_{2}\right) Q},
$$

and so $t_{1}+t_{2} \in G_{Q}(\mathcal{A})$. Thus $G_{Q}(\mathcal{A})$ is a group. It is closed, because whenever $t_{1}, t_{2}, \cdots \in G_{Q}(\mathcal{A})$ and $\lim t_{i}=t_{0}$, then for every operator $T \in \mathcal{A}$ and every natural $i$ we have

$$
e^{t_{i} Q} T e^{-t_{i}} \in \mathcal{A}
$$

and so

$$
e^{t_{0} Q} T e^{-t_{0} Q}=\lim _{i} e^{t_{i} Q} T e^{-t_{i} Q}
$$

is in $\mathcal{A}$, since $\mathcal{A}$ is closed. If $G_{Q}(\mathcal{A}) \neq\{0\}$ put

$$
\rho_{Q}(\mathcal{A})=\inf \left\{t>0: t \in G_{Q}(\mathcal{A})\right\},
$$

and $\rho_{Q}(\mathcal{A})=\infty$ if $t \in G_{Q}(\mathcal{A})$ for all positive $T$. We shall show that if $\rho_{Q}(\mathcal{A})>0$, then all algebras

$$
\mathcal{A}_{t}=e^{t Q} \mathcal{A} e^{-t Q}, \quad 0<t<\rho_{Q}(\mathcal{A})
$$

are different. Thus in this case there exist uncountably many copies of $\mathcal{A}$ and so the conclusion follows.

In fact, if $0<t_{1}<t_{2}<\rho_{Q}(\mathcal{A})$, then the relation $\mathcal{A}_{t_{1}}=\mathcal{A}_{t_{2}}$ implies $\mathcal{A}_{t_{2}-t_{1}}=\mathcal{A}$, i.e. $t_{2}-t_{1} \in G_{Q}(\mathcal{A})$, which is in contradiction with the fact that $0<t_{2}-t_{1}<$ $\rho_{Q}(\mathcal{A})$.

Observe now that if $\rho_{Q}(\mathcal{A})=0$, then $G_{Q}(\mathcal{A})=\mathbb{R}$. In fact, for each positive $\varepsilon$ there is a $t \in G_{Q}(\mathcal{A})$ with $0<t<\varepsilon$ such that $\mathcal{A}_{t}=\mathcal{A}$. Thus each interval of length $\varepsilon$ contains a $t$ belonging to $G_{Q}(\mathcal{A})$. That means that $G_{Q}(\mathcal{A})$ is dense in the real line, and, since it is closed, it equals to $\mathbb{R}$.

In order to prove our theorem it is sufficient to show, that for every $\mathcal{A} \in \mathfrak{A}(X)$ there is a $Q$ in $L(X)$, so that $\rho_{Q}(\mathcal{A})>0$, or, equivalently, $G_{Q}(\mathcal{A}) \neq \mathbb{R}$.

Assume towards contradiction that there is an $\mathcal{A}$ in $\mathfrak{A}(X)$ with $\rho_{Q}(\mathcal{A})=0$ for all $Q$ in $L(X)$ We fix this $\mathcal{A}$ from now on. Thus we have

$$
e^{Q} \mathcal{A} e^{-Q}=\mathcal{A}
$$

for all $Q$ in $L(X)$. Consequently

$$
e^{Q} T e^{-Q} T=T e^{Q} T e^{-Q}
$$

for all $T$ in $\mathcal{A}$ and all $Q$ in $L(X)$. Take a non-zero $Q$ satisfying $Q^{2}=0$ (it will be specified later). In this case, we have $e^{Q}=I+Q$ and $e^{-Q}=I-Q$. For such a $Q$ we can rewrite (3) as

$$
(I+Q) T(I-Q) T=T(I+Q) T(I-Q),
$$

for all $T$ in $\mathcal{A}$, which implies

$$
T Q T=\frac{1}{2}\left[Q T^{2}+T^{2} Q+T Q T Q-Q T Q T\right] .
$$


Multiplying both sides of (4) by $Q$ from the left and from the right, we obtain

$$
Q T Q T Q=0 .
$$

For obtaining desired contradiction we shall specify now $T$ and $Q$. Since $\operatorname{dim} X>$ 1, $\mathcal{A}$ must contain an operator $T$ which is not of the form $\lambda I$. For such an operator we can find an element $a$ in $X$ with $T a=b \neq \lambda a$ for all scalars $\lambda$. To see it assume towards contradiction that for some linearly independent $a, b$ in $X$ there are scalars $\lambda_{0}, \lambda_{1}$ and $\lambda_{2}, \lambda_{1} \neq \lambda_{2}$ with $T a=\lambda_{1} a, T b=\lambda_{2} b$ and $T(a+b)=\lambda_{0}(a+b)$. But this implies $\left(\lambda_{1}-\lambda_{0}\right) a+\left(\lambda_{2}-\lambda_{0}\right) b=0-$ a contradiction. We can find now a linear functional $f$ in $X^{*}$ with $f(a)=0$ and $f(b)=1$. Define $Q=f \otimes a(Q(x)=f(x) a)$. Thus $T Q(b)=b$, and so the value of the left-hand operator in (5) at the point $b$ equals to $a$. Since $a$ is different from zero, we obtain a contradiction with the formula (5). The conclusion follows.

The above theorem fails if we replace $L(X)$ by an arbitrary non-commutative unital Banach algebra. In [5] we constructed a unital Banach algebra which has only one infinite dimensional maximal commutative subalgebra, while the remaining ones are of dimension two. We do not know, however, the answer to the following question.

Problem 1. Let $A$ be a non-commutative unital Banach algebra. Does it contain an uncountable family of mutually isomorphic maximal commutative subalgebras?

The following question also seems to be of an interest.

Problem 2. Let $X$ be an infinite dimensional Banach space. Do there exist uncountably many maximal commutative subalgebras of $L(X)$ which are mutually non-isomorphic?

The above Problem is connected with the question the author was (long ago) asked by Aleksander Pełczyński: Let $X$ be a Banach space. How many different multiplications make of it a commutative unital Banach algebra?

It is well known (see [1]) that if $A$ is a commutative unital Banach algebra, then the set of operators

$$
\left\{T_{a} \in L(A): T_{a} x=a x, a, x \in A\right\}
$$

is a maximal commutative subalgebra of $L(A)$ which is clearly isomorphic with $A$. Thus all multiplications on $X$ making it a commutative unital Banach algebra are given by the family of all maximal commutative subalgebras of $L(X)$ which, as a Banach space, are isomorphic to $X$. With respect to this remark, we have

Proposition. Let $X$ be an infinite dimensional Banach space. Then there exist at least countably many mutually non-isomorphic maximal commutative subalgebras of $L(X)$, which, as the Banach spaces, are isomorphic to $X$. 
Proof. Choose a continuous linear functional $f$ on $X$ and an element $e \in X$ with $f(e)=1$. Put $Y=\operatorname{ker} f$. Then

$$
X=Y \oplus\{e\},
$$

where $\{e\}$ denotes the one-dimensional subspace of $X$ spanned by $e$. For an arbitrary $n \in \mathbb{N}$ decompose

$$
Y=Y_{n} \oplus Z_{n},
$$

where $\operatorname{dim} Y_{n}=n, Y_{n}=\operatorname{span}\left\{e_{1}, \ldots, e_{n}\right\}$. Making $Y_{n}$ a commutative Banach algebras under coordinatewise multiplication, and making $Z_{n}$ such an algebra under trivial (zero) multiplication, we made of $Y$ a non-unital commutative Banach algebra, whose unitization, as a Banach space, is isomorphic with $X$. Clearly, for different $n$ the obtained in this way multiplications on $X$ are non-isomorphic. The conclusion follows.

We could take different commutative multiplications on $Y_{n}$, but it would not help in answering to the Problem 2 , since for a fixed $n$ we have only finitely many such multiplications.

Returning to the Theorem, observe that its proof works neither for for locally convex spaces, nor for quasi-Banach (locally bounded) spaces. The reason is that for the locally convex spaces there can exist continuous linear operators not possessing exponentials (cf. [3]), while some quasi-Banach spaces do not possess continuous linear functionals $([4])$. In the case of locally pseudoconvex spaces (inverse limits of locally bounded spaces; for the locally bounded and locally pseudoconvex spaces the Reader is referred to [4]), there can occur rigid spaces i.e. topological vector spaces on which the only continuous linear operators are scalar multiples of the identity operator (see [2], or [4], pp 210-220). So if we want to have the above Theorem for the locally pseudoconvex spaces, we should replace condition $\operatorname{dim} X>1$ by the condition $\operatorname{dim} L(X)>1$.

\section{References}

[1] I.M. Gelfand, Normierte Ringe, Mat. Sb. 9 (1941), 3-24.

[2] N.J. Kalton, J.W. Roberts, A rigid subspace of $L_{0}$, Trans. Amer. Math. Soc. 266 (1981), 645-654.

[3] B. Mityagin, S. Rolewicz, W. Żelazko, Entire functions in $B_{0}$-algebras, Studia Math. 21 (1962), 291-306.

[4] S. Rolewicz, Metric Linear Spaces, PWN-Reidel, Warszawa, Dordrecht 1984.

[5] W. Żelazko, An infinite dimensional Banach algebra with all but one maximal abelian subalgebras of dimension two, Comm. Math. 48 (2008), 99-101.

Address: Wiesław Żelazko: Mathematical Institute, Polish Academy of Sciences, Śniadeckich 8, 00-956 Warsaw, Poland.

E-mail: zelazko@impan.pl

Received: 29 October 2014; revised: 10 March 2015 